Neurologische Abteilung, Landes Nerven Klinik, IgnazHarrer-Strasse 79, A-5020 Salzburg, Austria

M Huemer

V Niederweiser

G Ladurner

Correspondence to: Dr $M$ Huemer.

Received 13 April 1994 and in revised form 22 August 1994

Accepted 2 September 1994

\title{
Thrombolytic treatment for acute occlusion of the basilar artery
}

\author{
M Huemer, V Niederwieser, G Ladurner
}

\begin{abstract}
Sixteen patients with acute occlusion of the basilar artery were treated with systemic fibrinolysis. Recanalisation was achieved in 10 patients; five patients survived and 11 patients died. Survival was associated with vascular recanalisation in every case. Most of the survivors were younger than 50 . An age above 50 and a comatose state on admission seem to indicate a poor clinical outcome. The problem of multimorbidity in the older age group also affects outcome adversely. Haemorrhagic complications were found in two cases $(12 \cdot 5 \%)$, with clinical deterioration in one.
\end{abstract}

$(尹$ Neurol Neurosurg Psychiatry 1995;58:227-228)

Keywords: thrombolytic treatment; basilar artery occlusion; fibrinolysis

Zeumer et al first used topical thrombolysis for the treatment of acute basilar artery occlusion in 1982.12 Since then further reports have shown the clinical benefit of this approach..$^{3-7}$

We report, retrospectively, 16 patients who had acute occlusion of the basilar artery between 1985 and 1993 and who were treated by intravenous fibrinolysis.

\section{Patients and methods}

Twenty two patients with acute occlusion of the basilar artery were retrospectively investigated between 1985 and 1993.

Local intra-arterial thrombolysis was performed in six patients. These cases are not discussed further because of dissimilarities in technique and medication.

In 16 patients ( $11 \mathrm{men}$, five women) systemic thrombolytic treatment was given. These patients had a mean age of 62 (SD 14) years (range 31-78). In each case, $100 \mathrm{mg}$ tissue plasminogen activator was given over three hours.

Diagnosis was made clinically, followed by CT as soon as possible. In all our patients, initial CT showed no relevant abnormality.

To confirm the clinical diagnosis, angiography was performed in 13 of our patients as soon as possible (12 arterial angiography, one intravenous digital subtraction angiography) In eight patients, transcranial Doppler sonography (TCD) was performed before thrombolytic treatment. In three patients, TCD was the only means by which basilar artery occlusion was diagnosed.

Recanalisation was assessed by angiography in two cases and by TCD in 14 cases. In two patients, no assessment of recanalisation was performed because they survived less than 24 hours.

Recanalisation was considered to have occurred if angiography or TCD showed a patent basilar artery; recanalisation was regarded as not achieved if the vessel remained occluded, or if a high grade stenosis was found with relevant haemodynamic consequences.

The time from onset of symptoms to the start of the thrombolytic treatment was less than six hours in 13 patients, seven hours in one patient, and in one patient the period could not be assessed precisely from the case record.

Heparin was given to every patient after the thrombolytic procedure in a dosage (usually 800 to $1000 \mathrm{IU} / \mathrm{h}$ ) suitable to maintain the activated partial thromboplastin time at 1.5 to twice normal. In addition, conventional stroke treatment with hydroxyethyl starch (500 $\mathrm{ml}$ for five days) and intravenous pentoxifylline $(200 \mathrm{mg} /$ day $)$ was given routinely.

Computed tomography was performed after the thrombolytic treatment in every case, and repeated at intervals both routinely and with respect to clinical changes.

Clinical outcome was primarily assessed as survival and death. In the case of survival, outcome was regarded as not favourable if there was total dependence on nursing care, and as favourable, if independence from constant support was achieved.

\section{Results}

Sixteen patients aged between 31 and 78 had systemic thrombolytic treatment. Five patients survived, three with a favourable outcome. Eleven patients died. The patients who survived were younger than 51 , with the exception of a 74 year old man, who recovered well. In all survivors, recanalisation was accomplished. All 11 patients who died 
Systemic thrombolytic treatment in acute obstruction of the basilar artery

\begin{tabular}{lllll}
\hline \multicolumn{2}{l}{ Recanalisation $(n=10)$} & & \multicolumn{2}{l}{ No recanalisation $(n=6)$} \\
\cline { 5 - 5 } Survived & Died & & Survived & Died \\
\hline 5 & $5^{\star}$ & 0 & 6 \\
\hline
\end{tabular}

* Three patients with severe non-cerebral complications (myocardial infarction, urosepsis, cardiac failure).

were older than 50 . In six of them, recanalisation was achieved (table).

Recanalisation was achieved in 10 patients $(62 \%)$, five of whom survived. All six patients in whom recanalisation could not be achieved died (table). In the subgroup older than 50, recanalisation was achieved in seven out of 13 cases $(54 \%)$.

Three of the five patients who died after recanalisation had severe non-cerebral complications that led to death after appreciable recovery from their initial neurological deficits. A 59 year old woman died on day three from acute myocardial infarction. A 53 year old man died of acute cardiac failure on day eight; cor bovinum and hepatic cirrhosis were present at necropsy. A 66 year old man died 33 days after thrombolytic treatment as a result of urosepsis based on a pre-existing chronic pyelonephritis.

Twelve patients were comatose on admission. In six of these patients recanalisation was achieved. Only two of the six recanalised patients survived.

Recanalisation was accomplished in the four patients who were not comatose on admission and three of them survived.

Bleeding complications occurred, with major intracerebral haemorrhages in two patients $(12.5 \%)$. In a 43 year old man, a haemorrhage was found in the frontoparietal region of the right hemisphere by routine CT. The haemorrhage did not affect a good clinical outcome. A pontine haemorrhage occurred in a 78 year old woman, who died on day 16 after thrombolysis in an unresolved comatose state. Brain necropsy showed pronounced atherosclerotic changes in the basal brain vessels.

\section{Discussion}

Despite the few patients and sparse reports in the literature it is considered that lysis induced recanalisation of the basilar artery can be of benefit to the patient. Systemic thrombolysis-first described in an encouraging case report by Wildemann et al, ${ }^{4}$ and subsequently by von Kummer et $a l^{5}$ with a poor outcome in four of five patients-has not been widely used as a therapeutic approach.

In our series of 16 patients, the outcome seems to be dependent on recanalisation of the occluded vessel, as previously reported.
Recanalisation is mandatory for survival, although it does not guarantee it; nonrecanalisation is inevitably correlated with bad clinical outcome ( $100 \%$ died).

Our experience indicates that survival of a patient older than 50 is a rare event, even if recanalisation is achieved. Nevertheless, in seven of $13(54 \%)$ patients older than 50 recanalisation was achieved.

It is important to note that of the five patients who died despite recanalisation, three had severe non-cerebral complications. If these non-cerebral causes of death are excluded, the success rate improves with eight survivors $v$ two cerebral causes of death in those patients with recanalisation. Thus our data suggest that poor clinical outcome, especially in elderly patients, is more often due to concomitant medical illness (three of five $(60 \%))$.

Moreover, in patients with initial coma, recanalisation is less likely to be achieved (only $50 \%$ in our series) and survival is less probable even with recanalisation.

A comparison of our survival rate (31\%) and favourable survivals (19\%) shows similar figures to those of Hacke $e t a l^{3}$ for a topical thrombolytic procedure (33\% survival, $23 \%$ favourable). Our recanalisation rate of $62 \%$ is higher than that of Hacke et al, (44\%), ${ }^{3}$ equals that of Pfeiffer et $a l^{6}{ }^{6}$ and is less than that of Moebius et al (78\%). ${ }^{7}$ Our haemorrhagic complication rate of $12.5 \%$ slightly exceeds that given by Hacke et al (9\%). ${ }^{3}$

In conclusion, we believe that systemic thrombolytic treatment is a valuable tool for treating acute occlusion of the basilar artery. The results seem comparable with those achieved by a topical thrombolysis. Although outcome in patients younger than 50 seems most promising, its application should not be withheld from older patients, for benefit may result, especially in the absence of initial coma and concomitant medical illness.

1 Zeumer H, Hacke W, Kolmann HL, Poeck K. Lokale Fibrinolyse bei Basilaristhrombose. Dtsch Med Wochenschr 1982;107:728-31.

2 Zeumer H, Hacke W, Ringelstein EB. Local intraarterial thrombolysis in vertebrobasilar thrombembolic disease. Am $\mathcal{F}$ Neuroradiol 1983;4:401-4.

3 Hacke W, Zeumer H, Ferbert A, Brückmann H, del Zoppo GJ. Intra-arterial thrombolytic therapy improves Zoppo GJ. Intra-arterial thrombolytic therapy improves outcome in patients with acute vert
disease. Stroke 1988;19:1216-22.

4 Wildemann $B$, Hutschenreuter $M$, Krieger $D$, von Kummer R. Infusion of recombinant tissue plasminogen activator for treatment of basilar artery occlusion. Stroke 1990;21:1513-4.

5 von Kummer $R$, Forsting $M$, Sartor $K$, Hacke W. Intravenous recombinant tissue plasminogen activator in acute stroke. In: Hacke W, del Zoppo GJ, Hirschberg $\mathrm{M}$, eds. Thrombolytic therapy in acute ischemic stroke. New York: Springer, 1991:161-7.

6 Pfeiffer G, Thayssen G, Arlt A, Siepmann G, Zeumer H, Kunze K. Vertebrobasilar occlusion: outcome with and without local intraarterial fibrinolysis. In: Hacke W, del Zoppo GJ, Hirschberg M, eds. Thrombolytic therapy in Zoppo GJ, Hirschberg M, eds. Thrombolytic therapy in

7 Möbius E, Berg-Dammer E, Kühne D, Nahser HC. Local thrombolytic treatment in acute basilar artery occlusion: thrombolytic treatment in acute basilar artery occlusion: experience with 18 patients. In: Hacke W, del Zoppo GJ, Hirschberg M, eds. Thrombolytic therapy in 